\title{
Character Agents in E-Learning Interface Using Multimodal Real-Time Interaction
}

\author{
Hua Wang ${ }^{2}$, Jie Yang ${ }^{1}$, Mark Chignell ${ }^{2}$, and Mitsuru Ishizuka ${ }^{1}$ \\ ${ }^{1}$ University of Tokyo \\ midi.jie.yang@gmail.com, \\ ishizuka@miv.t.y-tokyo.ac.jp \\ ${ }^{2}$ University of Toronto \\ hwang@mie.utoronto.ca, \\ chignel@mie.utoronto.ca
}

\begin{abstract}
This paper describes an e-learning interface with multiple tutoring character agents. The character agents use eye movement information to facilitate empathy-relevant reasoning and behavior. Eye Information is used to monitor user's attention and interests, to personalize the agent behaviors, and for exchanging information of different learners. The system reacts to multiple users' eye information in real-time and the empathic character agents owned by each learner exchange learner's information to help to form the online learning community. Based on these measures, the interface infers the focus of attention of the learner and responds accordingly with affective and instructional behaviors. The paper will also report on some preliminary usability test results concerning how users respond to the empathic functions and interact with other learners using the character agents.
\end{abstract}

Keywords: Multiple user interface, e-learning, character agent, tutoring, educational interface.

\section{Introduction}

Learners can lose motivation and concentration easily, especially in a virtual education environment that is not tailored to their needs, and where they may be little contact with live human teachers. As Palloff and Pratt [1] noted "the key to success in our online classes rests not with the content that is being presented but with the method by which the course is being delivered". In traditional educational settings, good teachers recognized learning needs and learning styles and adjusted the selection and presentation of content accordingly.

In online learning there is a need to create more effective interaction between e-learning content and learners. In particular, increasing motivation by stimulating learner's interest is important. A related concern is how to achieve a more natural and friendly environment for learning. We will address this concern by detecting the attention information from the real-time eye tracking data from each learner and modify instructional strategies based on the different learning patterns for each learner. 
Eye movements provide an indication of learner interest and focus of attention. They provide useful feedback to character agents attempting to personalize learning interactions. Character agents represent a means of bringing back some of the human functionality of a teacher. With appropriately designed and implemented animated agents, learners may be more motivated, and may find learning more fun. However, amusing animations in themselves may not lead to significant improvement in terms of comprehension or recall. Animated software agents need to have intelligence and knowledge about the learner, in order to personalize and focus the instructional strategy.

Figure 1 shows a character agent as a human-like figure embedded within the content on a Web page. In this paper, we use real time eye gaze interaction data as well as recorded study performance to provide appropriate feedback to character agents, in order to make learning more personalized and efficient. This paper will address the issues of when and how such agents with emotional interactions should be used for the interaction between learners and system.

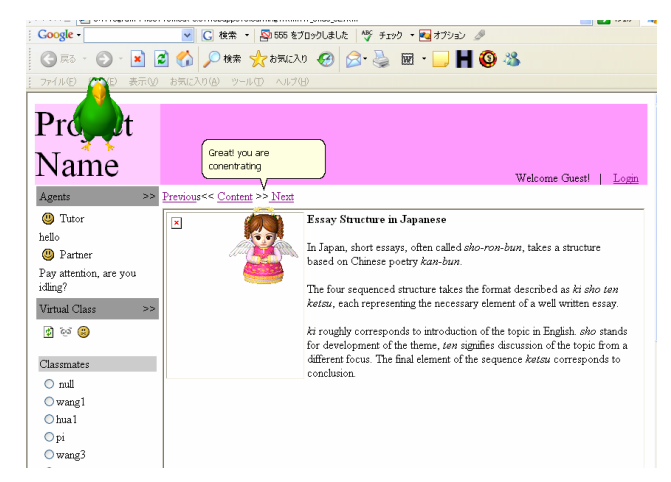

Fig. 1. Interface Appearance 2 Related Work

Animated pedagogical agents can promote effective learning in computer-based learning environments. Learning materials incorporating interactive agents engender a higher degree of interest than similar materials that lack animated agents If such techniques were combined with animated agent technologies, it might then be possible to create an agent that can display emotions and attitudes as appropriate to convey empathy and solidarity with the learner, and thus further promote learner motivation. [2] Fabri et al. [3] described a system for supporting meetings between people in educational virtual environments using quasi face-to-face communication via their character agents. In other cases, the agent is a stand-alone software agent, rather than a persona or image of an actual human. Stone et al. in their COSMO system used a life-like character that teaches how to treat plants [4].

Recent research in student modeling has attempted to allow testing of multiple learner traits in one model [5]. Each of these papers introduces a novel approach towards testing multiple learner traits. Nevertheless, there is not much work on how to make the real-time interaction among different learners, as well as how to interact among different learners with non-verbal information of each learner. 
Eye tracking is an important tool for detecting users' attention information and focus on certain content. Applications using eye tracking can be diagnostic or interactive. In diagnostic use, eye movement data provides evidence of the learner's focus of attention over time and can be used to evaluate the usability of interfaces [6] or to guide the decision making of a character agent. For instance, Johnson [7] used eye-tracking to assist character agents during foreign language/culture training. In interactive use of eye tracking for input, a system responds to the observed eye movements, which can serve as an input modality [8].

For the learner's attention information and performance, the ontology base is used to store and communicate learners' data. By using ontology, the knowledge base provides information, both instant and historical, for Empathic tutor virtual class and also the instant communication between agents. Explicit Ontology is easy and flexible to control the character agent.

\section{Education Interface Structure}

Broadly defined, an intelligent tutoring system is educational software containing an artificial intelligence component. The software tracks students' work, tailoring feedback and hints along the way. By collecting information on a particular student's performance, the software can make inferences about strengths and weaknesses, and can suggest additional work. Our system differs in the way that the interface use real-time interaction with learners resembles the real learning process with teachers) and interact with learners' attention. Figure 2 shows a general system diagram of the system.

In the overview diagram of our system, the character agents interact with learners, exhibiting emotional and social behaviors, as well as providing instructions and guidance to learning content. In addition to input from the user 's text input, input timing, and mouse movement information, etc., feedback about past performance and behavior is also obtained from the student performance knowledge base, allowing agents to react to learners based on that information.

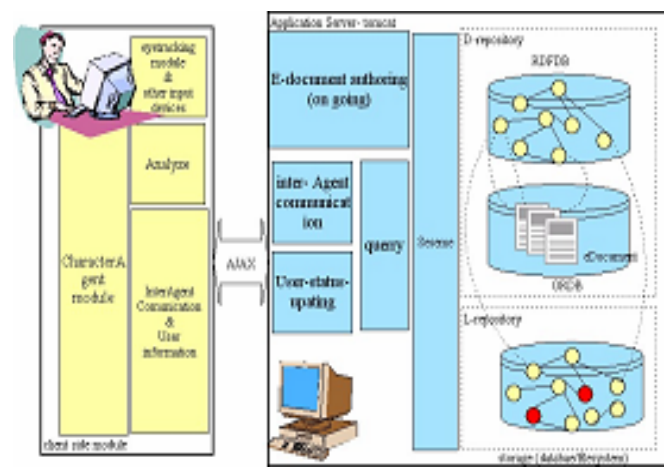

Fig. 2. The system structure 
For the multiple learner communication, the system use each learner' character agent to exchange learners' interests, attention information. Each learner has a character agent to represent himself. Besides, each learner's motivation is also linked in the learning process. When another learner has information to share, his agent will come up and pass the information to the other learner. During the interaction among learners, agents detect learner's status and use multiple data channels to collect learner's information such as movements, keyboard inputs, voice, etc.

The functions of character agent can be divided into those involving explicit or implicit outputs from the user, and those involving inputs to the user. In case of outputs from the user, empathy involves functions such as monitoring emotions and interest. In terms of output to the user, empathy involves showing appropriate emotions and providing appropriate feedback concerning the agent's understanding of the users' interests and emotions. Real time feedback from eye movement is detected by eye tracking, and the character agents use this information to interact with learners, exhibiting emotional and social behaviors, as well as providing instructions and guidance to learning content. Information about the learner's past behavior and interests based from their eye tracking data is also available to the agent and supplements the types of feedback and input.

The interface provides the multiple learner environments. The information of each learner is stored with ontology base and the information is sent to character agents. The character agents share the information of the learners according to their learning states. In our learning model, the information from learners is stored in the knowledge base using ontology. Ontology system contains learners' performance data, historical data, and real-time interaction data, etc. in different layers and agents can easily access these types of information and give the feedback to learners.

\subsection{Real-Time Eye Gaze Interaction}

Figure 3 shows how the character agent reacts to feedback about the learner's status based on eye-tracking information. In this example, the eye tracker collects eye gaze information and the system then infers what the learner is currently attending to. This

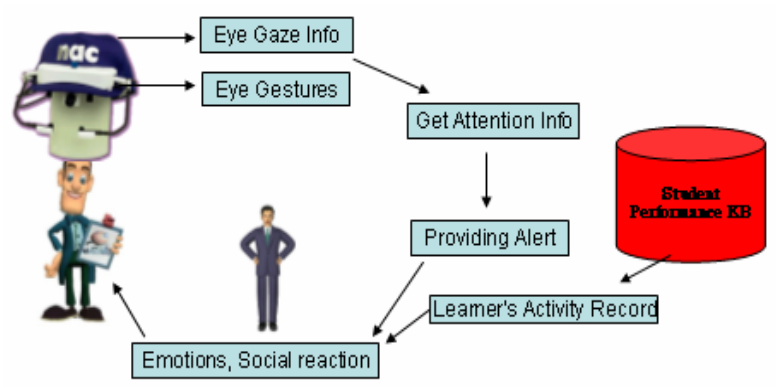

Fig. 3. Real-Time Use of Eye Information in ESA 
information is then combined with the learner's activity records, and an appropriate pre-set strategy is selected. The character agent then provides feedback to the learner, tailoring the instructions and emotions (e.g., facial expressions) to the situation.

\subsection{Character Agent with Real-Time Interaction}

In our system, one or more character agents interact with learners using synthetic speech and visual gestures. The character agents can adjust their behavior in response to learner requests and inferred learner needs. The character agents perform several behaviors including the display of different types of emotion. The agent's emotional response depends on the learner's performance. For instance, an agent shows a happy/satisfied emotion if the learner concentrates on the current study topic. In contrast, if the learner seems to lose concentration, the agent will show mild anger or alert the learner. The agent also shows empathy when the learner is stuck. In general, the character agent interacts between the educational content and the learner. Other tasks of a character agent include explaining the study material and provide hints when necessary, moving around the screen to get or direct user attention, and to highlight information.

The character agents are "eye-aware" because they use eye movements, pupil dilation, and changes in overall eye position to make inferences about the state of the learner and to guide his behavior. After getting learner's eye position information and current area of interest or concentration, the agents can move around to highlight the current learning topic, to attract or focus the learner's attention. For instance, with eye gaze data, agents react to the eye information in real time through actions such as moving to the place being looked at, or by showing the detailed information content for where learners are looking at, etc. ESA can also accommodate multimodal input from the user, including text input, voice input and eye information input, e.g., choosing a hypertext link by gazing at a corresponding point of the screen for longer than a threshold amount of time.

\section{Implementation}

The system uses server-client communication system to build the multiple learner interface JavaScript and AJAX are used to build the interactive contents which can get real time information and events from learner side. The eye gaze data is stored and transferred using an XML file. The interface uses a two-dimensional graphical window to display character agents and education content. The graphical window interface shows the education content, flash animations, movie clips, and agent behaviors. The Eye Marker eye tracking system was used to detect the eye information and the basic data was collected using 2 cameras facing towards the eye. The learners' information is stored in the form of ontology using RDF files (Figure 4) and the sturdy relationship between different learners can be traced using the knowledge base (Figure 5).

The knowledge base using Ontology is designed and implemented by protégé [9]. Ontology provides the controlled vocabulary for learning domain. 


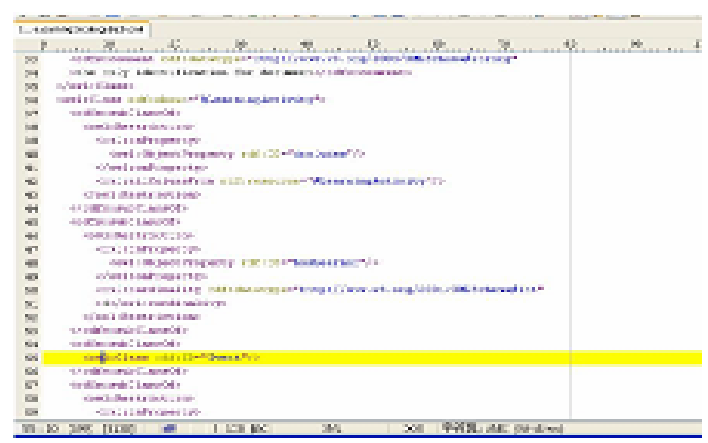

Fig. 4. Learner's Information stored in RDF files

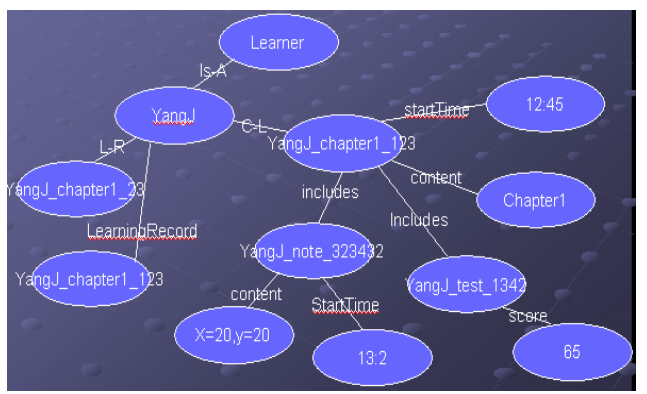

Fig. 5. Relationship among different learners

\section{Overall Observations}

We carried the informal experiences using the interface after we implement the system. In a usability study, 8 subjects participated using the version of the multiple learners support interface. They learned two series of English lesson. Each learning session lasted about 45 minutes. After the session, the subjects answered questionnaires and commented on the system.

We analyzed the questionnaires and comments from the subjects. Participants felt that the interactions among the learners made them more involved in the learning process. They indicated that the information about how others are learning made them feel more involved in the current learning topic. They also indicated that they found it is convenient to use the character agent in sharing their study information among other learners, which makes them feel comfortable.

Participants in this initial study said that they found the character agents useful and that they listened to the explanation of contents from the agents more carefully than if they had been reading the contents without the supervision and assistance of the character agent. During the learning process, character agents achieve a combination of informational and motivational goals simultaneously during the interaction with learners. For example, hints and suggestions were sometimes used from getting the learners' attention information about what the learner wants to do. 


\section{Discussions and Future Work}

By using the character agents for multiple learners, each learner can get other learning partner's study information, interest, thus they can find the learning partners with similar learning backgrounds and interact with each other. By getting information about learner response, character interfaces can interact with multiple learners more efficiently and provide appropriate feedback. The different versions of character agents are used to observe the different roles in the learning process. In the system, the size, voice, speed of the speech, balloon styles, etc. can be changed to meet different situations. Such agents can provide important aspects of social interaction when the student is working with e-learning content. This type of agent-based interaction can then supplement the beneficial social interactions that occur with human teachers, tutors, and fellow students within a learning community.

Aside from an explicitly educational context, real-time eye gaze interaction can be used in Web navigation. By getting what part of users are more interested in, the system can provide real time feedback to users and help them to get target information more smoothly.

\section{References}

1. Palloff, R.M., Pratt, K.: Lessons from the cyberspace classroom: The realities of online teaching. Jossey-Bass, San Francisco (2001)

2. Klein, J., Moon, Y., Picard, R.: This computer responds to learner frustration: Theory, design, and results. Interacting with Computers, 119-140 (2002)

3. Fabri, M., Moore, D., Hobbs, D.: Mediating the Expression of Emotion in Educational Collaborative Virtual Environments: An Experimental Study. Virtual Reality Journal (2004)

4. Stone, B., Lester, J.: Dynamically Sequencing an Animated Pedagogical Agent. In: Proceedings of the 13th National Conference on Artificial Intelligence, Portland, OR, pp. 424-431 (August 1996)

5. Welch, R.E., Frick, T.W.: Computerized adaptive testing in instructional settings. Educational Technology Research and Development 41(3), 47-62 (1993)

6. Duchowski, T.: Eye Tracking Methodology: Theory and Practice. Springer, London, UK (2003)

7. Johnson, W.L., Marsella, S., Mote, H., Vilhjalmsson, S., Narayanan, S., Choi, S.: Language Training System: Supporting the Rapid Acquisition of Foreign Language and Cultural Skills

8. Faraday, P., Sutclie, A.: An empirical study of attending and comprehending multimedia presentations. In: Proceedings of ACM Multimedia, pp. 265-275. ACM Press, Boston, MA (1996)

9. http://protege.stanford.edu 